\title{
Correction to: The Impact of Social Capital and Media Use on the Political Participation of Urban Residents
}

\author{
Fanbin Zeng ${ }^{1} \cdot$ Chen Yuqi $^{2}$ \\ Published online: 6 November 2018 \\ (C) Springer Nature B.V. 2018
}

\section{Correction to: East Asia \\ https://doi.org/10.1007/s12140-018-9284-7}

The original publication contained an error. The second author Chen Yuqi was not included by mistake.

Fanbin Zeng

zengfanbin@vip.sina.com

$\triangle$ Chen Yuqi

413185569@qq.com

1 School of Journalism and Communication, Jinan University Guangzhou, Guangzhou 510632, China

2 International Journalism, International School, Jinan University, No.601, West Huangpu Avenue, Tianhe District, Guangzhou, Guangdong 510632, People's Republic of China 